\title{
Il ruolo delle risorse intangibili nella letteratura manageriale. Principali linee di ricerca e analisi future $^{1}$
}

\section{Cinzia Dessì - Michela Floris}

\begin{abstract}
Obiettivo del paper: Il presente lavoro si propone di realizzare una review della letteratura sul tema delle risorse intangibili per delinearne un modello interpretativo. Metodologia: La metodologia utilizzata è la narrative review.

Risultati: I risultati evidenziano: a. l'inesistenza di un'univoca definizione di "risorse intangibili", b. le teorie maggiormente utilizzate, c. i principali "filoni" di studio, $d$. nuovi ambiti di ricerca verso i quali orientare futuri studi.

Limiti della ricerca: I limiti sono principalmente riconducibili ai database utilizzati.

Implicazioni pratiche: Le implicazioni sono di duplice natura. Dal punto di vista accademico si contribuisce allo stato dellarte su tale tematica proponendo ulteriori sviluppi futuri; dal punto di vista manageriale si indirizza l'analisi dell'imprenditore e del consulente verso quelle risorse che contribuiscono alle performance aziendali.

Originalità del lavoro: Il lavoro si inserisce nel dibattito accademico sulle risorse con una review della letteratura che copre un periodo di tempo abbastanza lungo (1990-2017), concentrandosi sulla letteratura che utilizza la locuzione "risorse intangibili" come un "umbrella concept" senza soffermarsi esclusivamente su alcune fattispecie di risorse e abbracciando tutta la letteratura manageriale senza circoscrivere l'analisi a specifiche aree di studio.
\end{abstract}

Parole chiave: risorse intangibili; review della letteratura; narrative review

Purpose of the paper: This research aims to realize a literature review about intangible resources in managerial studies, in order to conceive an interpretive model.

Methodology: Narrative review.

Findings: Results show that a unique definition of intangible resources does not exist. In addition, findings underline the most used theories, the main streams of research and suggest avenues for future studies.

Limitations: Limitations depends on databases used.

Implications: Implications are twofold. We firstly contribute to the literature offering new research streams, and secondly the research helps practitioners identifying intangible resources that represent the core to pursue the competitive advantage.

Originality of the paper: This paper offers an in depth analysis of the managerial literature on intangible resources and draws the state of the art suggesting future perspectives of analysis. It covers a very large time span (1990-2017), it concentrates on contributions that have used "intangibles resources" as an "umbrella concept", and it focus on the whole of managerial literature (i.e. not only financial or accounting).

Key words: intangible resources; literature review ; narrative review

1 Il lavoro è frutto del lavoro congiunto dei due autori. Tuttavia i par. 1, 3 e 6 sono attribuibili a Cinzia Dessì e i par. 2, 4 e 5 a Michela Floris. 


\section{sinergie}

Vol. 35, N. 103, 2017

\section{Introduzione}

Lattuale contesto socio-economico impone alle imprese una revisione delle proprie strategie (Yannopoulos, 2011) al fine di individuare, sviluppare e mantenere nel tempo un sistema di risorse uniche a fondamento di un duraturo e sostenibile vantaggio competitivo (Brondoni, 2002; Jones e Kierzkowski, 2003; Gottfredson e Aspinall, 2005; Harris, 2005).

Tale circostanza è supportata da note teorie, prima tra tutte la Resource Based View (RBV), che considera l'impresa come un sistema di risorse strategiche, tangibili e intangibili atte a sostenere nel tempo il proprio vantaggio competitivo (Penrose, 1959; Wernerfelt, 1984; Barney, 1991). Tra queste risorse assumono un valore fondamentale le cosiddette risorse intangibili (RI) (Hall, 1992; 1993), ovvero quelle specifiche risorse che per la loro stessa natura non possono essere cedute o trasferite e non sono facilmente imitabili e sostituibili. In quest'ottica, le risorse intangibili rappresentano il fattore chiave per il perseguimento di un vantaggio competitivo, su cui le imprese dovrebbero puntare per conseguire performance elevate (Barney, 1991).

Alla luce di queste considerazioni il presente lavoro, attraverso una narrative review della letteratura manageriale, ha l'obiettivo di individuare le principali definizioni esistenti in tema di risorse intangibili e analizzare i principali aspetti sui quali la letteratura manageriale si è soffermata, al fine di proporre un modello interpretativo del tema. Limiti e implicazioni della ricerca sono discusse nella parte finale.

Inoltre, la crescente rilevanza acquisita dall'analisi delle RI ha imposto il crescente ricorso ad approcci teorici basati sulle risorse. Tra le varie teorie ve ne sono alcune che ancora oggi rappresentano un punto di riferimento nella letteratura, in particolare: la Resource Based View (Wernerfelt, 1984; Barney, 1991), la Knowledge Based View (Grant, 1996; Nonaka et al., 2000) e la teoria delle Dynamic Capabilities (Teece et al., 1997). Le citate teorie, insieme ad altre meno utilizzate, verranno discusse successivamente.

\section{Metodologia}

La metodologia utilizzata è la review della letteratura (Petticrew e Roberts, 2008). In relazione all'oggetto di analisi, possono essere utilizzate diverse literature review (Cooper, 1998) come: la case research syntheses (Gray et al., 2004; Melville et al., 2004) che include i risultati empirici delle ricerche, la theoretical review (Musiolik et al., 2012; Atherton, 2013), che evidenzia solo le teorie e i contributi alla creazione di nuova teoria, la meta-analysis review (Rubera e Kirca, 2012) che presenta i risultati di precedenti ricerche e li analizza nuovamente attraverso tecniche statistiche e la descriptive o narrative review (Cooper, 1998) grazie alla quale i dati di natura quantitativa vengono riassunti, combinati, integrati e interpretati.

In questa sede si ritiene opportuno il ricorso alla narrative literature review (Baumeister e Leary, 1997; Dixon-Woods et al., 2006) poiché essa consente di: a) descrivere lo stato dell'arte di una ben definita tematica; b) individuare nuove dimensioni di analisi non presenti nella letteratura esistente; c) fornire un'analisi critica dei contributi oggetto dell'analisi (Rumrill Jr e Fitzgerald, 2001). Oltre a ciò, diversi lavori hanno utilizzato 
tale metodologia per studiare le RI (Cañibano et al., 2000; Kaufmann e Schneider, 2004). In altri termini, il presente contributo, in linea con quanto evidenziato da MacInnis (2011), si propone come paper concettuale che non solo riassume ("summarizing") lo stato dell'arte di un filone di studi ma tenta di creare un "integrative framework" per rendere organici i contributi e proporre un modello interpretativo che colga quanto già pubblicato, suggerendo inoltre interessanti spunti di riflessione per le ricerche future.

Attraverso la narrative review si intende analizzare la letteratura manageriale esistente in tema di risorse intangibili dal 1990 al 2017 (mese di aprile), disponibile nei database ISI Web of Knowledge Thomson Reuters congiuntamente al motore di ricerca Google Scholar. Per la scelta dell'arco temporale ci si è riferiti a quanto suggerito da Petticrew e Roberts (2008) che individuano nella soggettività del ricercatore il principale punto di partenza per una review della letteratura. La scelta di cominciare l'analisi dal 1990 è dettata dal fatto che i seminal articles sono stati pubblicati a partire da quel periodo e questa circostanza consente di delineare il trend evolutivo di tale filone di studi fino ai giorni nostri. Relativamente alla selezione dei database, il primo copre circa 9300 riviste scientifiche di differenti discipline ad elevato impatto scientifico, mentre il secondo consente di individuare gli studi e la produzione scientifica di un determinato fenomeno evidenziando i risultati più pertinenti alla ricerca nelle prime pagine.

Lo studio si è sviluppato in quattro fasi. (I) Individuazione degli articoli pubblicati su riviste ad elevato impact factor e censite in ISI Web of Knowledge. (II) Individuazione degli articoli inseriti nel database Google Scholar. (III) Combinazione dei risultati ottenuti delle due precedenti fasi; (IV) analisi del contenuto degli articoli e discussione dei risultati.

Relativamente alla prima fase della ricerca, i documenti presi in considerazione sono in lingua inglese e relativi alla fattispecie scientifica più selettiva "article". La parola chiave usata è "intangible resource/s" poiché: a. alcune meta - analytic review mettono in evidenza come nella letteratura vi sia la tendenza ad utilizzare il termine "intangible resource" come un "umbrella concept" al fine di descrivere un gruppo omogeneo di risorse (Crook et al., 2008); b. in differenti contributi scientifici (Diefenbach, 2006) oggetto dell'analisi, molti autori affermano di utilizzare la locuzione "intangible resources" riferendosi indistintamente ai concetti di capacità, competenze distintive o conoscenza (Villalonga, 2004).

La ricerca ha prodotto 198 risultati, ulteriormente selezionati nel modo seguente: 1 . circoscrivendo alle sole categorie "Management" e "Business" 2. eliminando i contributi che contengono la parola chiave in maniera meramente citazionale. Al termine di questo step, i contributi presi in considerazione sono stati 83 . Non sono stati considerati ulteriori discriminanti per poter avere un quadro il più possibile esaustivo. La scelta, ad esempio, di utilizzare altre metodologie come la citation analysis avrebbe distorto dall'obiettivo principale del lavoro e soprattutto non avrebbe consentito l'analisi dei contributi più recenti.

Per tale motivo, si è proceduto ad ampliare il numero degli articoli da analizzare, attraverso la seconda fase della ricerca, ossia l'interrogazione del motore di ricerca "Google Scholar" (Meho e Yang, 2007). In questo modo sono stati consultati ulteriori 16 articoli pubblicati su riviste scientifiche aventi Impact Factor, ma non censite in ISI Web of Knowledge. Quattro
Cinzia Dessì

Michela Floris

Il ruolo delle risorse

Intangibili nella letteratura

manageriale. Principali linee

di ricerca e analisi future 
sinergie Vol. 35, N. 103, 2017

articoli sono stati eliminati in quanto già emersi nella fase precedente. Dalla terza fase, dunque emerge il risultato definitivo pari a 12 che sommato al risultato precedente definisce in 95 lavori il numero di paper da analizzare. La quarta fase rappresenta l'analisi e l'interpretazione del contenuto degli articoli ed è discussa nella sezione successiva.

\section{Analisi dei risultati}

Il primo aspetto che emerge dall'analisi dei 95 articoli è che la maggioranza di essi fa riferimento ai contributi di Hall (1992; 1993), Barney $(1991 ; 1997 ; 2001)$, Porter $(1985 ; 1991)$ e Penrose (1959). Alla luce di questa considerazione generale, il contenuto specifico è stato analizzato attraverso un coding (Saldaña, 2012) che ha tenuto conto dei seguenti elementi:(A) Parole chiave e definizione di RI; (B) Analisi dell'obiettivo principale del paper; (C) Teoria di riferimento e metodologia utilizzata; (D) Analisi del Focus, ossia aspetto centrale sul quale gli autori hanno indirizzato la loro attenzione.

\subsection{A) Analisi delle parole chiave e definizione di RI}

Dallanalisi condotta emerge l'inesistenza di un unico termine per identificare e qualificare le RI, la cui locuzione viene utilizzata come un "umbrella concept" comprendente espressioni come: intangible resources, intangible assets, intellectual capital, knowledge assets, organizational assets, ecc. Quasi la totalità degli articoli analizzati poggia i propri contributi sulla definizione proposta da Hall (1993, p. 608) che qualifica come RI "the intellectual property rights of patents, trademarks, copyright and registered designs; trade secrets; contracts and licenses; data bases; information in the public domain; personal and organizational networks; the know-how of employees, professional advisers, suppliers and distributors; the reputation of products and company; the culture of the organization". Lautore inoltre distingue tali aspetti in cosiddetti assets e competenze ("may be classified as 'assets' or 'competencies"') e considera tra le competenze la cultura organizzativa e il know-how, mentre i restanti aspetti vengono classificati come assets. Individua il know-how come risorsa distintiva propria dellorganizzazione, che si manifesta in relazione alle abilità e all'esperienza dei dipendenti. Secondo Hall (1993), la cultura organizzativa è data dalle credenze, dalla conoscenza, dagli atteggiamenti mentali e dagli usi a cui gli individui sono esposti; e riflette abitudini, pensieri, valori e attitudini dei soggetti.

La definizione proposta da Hall è stata ripresentata anche nei lavori di Crystal X Jiang et al. (2011), Moustaghfir (2009), Harvey e Lusch (1995). Norman et al. (2013) i quali sostengono che le RI sono quelle risorse che non possono essere facilmente quantificabili, ad esempio la reputazione o il brand. Diversamente, alcuni autori partono dalla concezione del capitale intellettuale, distinguendo lo stesso in alcuni gruppi, o approfondendo la sua diffusione in alcuni contesti specifici (Zambon et al., 2016). Nello specifico, Kianto et al. (2013) definiscono l'intellectual capital (IC) come la conoscenza e la gestione di essa, i cui principali drivers sono il capitale umano, strutturale, le reti di relazioni e la gestione delle stesse 
risorse. Hayton (2005, p. 140) definisce il capitale intellettuale come " $a$ bundle of organizational resources comprised of human capital, intellectual property, and reputational capital that are tangible and intangible in nature and can be leveraged o create value", qualificandolo come fonte di tre differenti assets: capitale umano, proprietà intellettuale e capitale reputazionale, ritenendo invece che il capitale strutturale in realtà sembri destare confusione nella qualificazione dello stesso. Lautore inoltre ritiene opportuno distinguere la conoscenza posseduta, cosiddetta "embodied" e l'abilità dell'organizzazione di combinare la conoscenza al fine di generare valore. Bontis et al. (1999) presentano una review sugli strumenti di misurazione delle RI, identificando quattro differenti strumenti: human resource accounting, balanced scorecard, economic value added intellectual capital. Essi ritengono che il capitale intellettuale sia costituito da tutte le RI, includendo il capitale umano distinto in competenze, attitudini e agilità intellettuale e il capitale strutturale costituito dalle relazioni, dallorganizzazione (struttura, cultura, processi e routines) e dal rinnovo e sviluppo (progetti di R\&D, nuovi prodotti). Anche Pike et al. (2005) affermano che le RI sono costituite da tre grandi gruppi, capitale umano, organizzativo e relazionale, contestualizzando l'identificazione di tali risorse alle R\&D organizations. Aragón Sánchez e Sánchez Marín (2005) identificano alcuni "intangible factors" che più di altri permettono alle imprese di piccole e medie dimensioni di perseguire un vantaggio competitivo, ossia il cambiamento strutturale ed organizzativo, le risorse umane, le risorse tecnologiche e l'innovazione. Carmeli (2004) afferma che le RI hanno un ruolo fondamentale nella creazione del valore e le definisce come tutto ciò che di fatto non si presenta nei bilanci, come la cultura organizzativa, la reputazione e il controllo interno. Fernández et al. (2000) definiscono le RI come quelle risorse che si riferiscono alla conoscenza o all'informazione e riprendono la classificazione formulata da Hall (1992; 1993).

Le definizioni sono accomunate dalla rilevanza del capitale umano e della conoscenza come fattori chiave e distintivi. Le risorse umane rappresentano l'elemento cardine da cui derivano lo sviluppo della conoscenza, il perseguimento di un vantaggio sostenibile e l'attivazione di processi di crescita interna.

\subsection{B) Analisi dell'obiettivo del paper}

La totalità dei lavori scientifici analizzati è concorde sul fatto che le imprese devono essere capaci di individuare e isolare le proprie RI per poterle porre alla base del proprio vantaggio competitivo (Porter, 1985) e sopravvivere a lungo nel mercato (D’Aveni e Gunther, 1995).

Dai 95 articoli selezionati emerge che l'obiettivo del paper si può incardinare in uno dei seguenti raggruppamenti:

(b.1) Definire e classificare le RI (Hall, 1992; 1993; Harvey e Lusch, 1995; Bontis et al., 1999; Fernández et al., 2000; Carmeli, 2004; Galbreath, 2005; Gardner, 2005; Hayton, 2005; Rodriguez e Rodríguez, 2005; Zyglidopoulos, 2005; Diefenbach, 2006; Hewitt-Dundas, 2006; Marcus e Anderson, 2006; Bontis et al., 2007; Cepeda e Vera, 2007; Camisón e Villar, 2009; Carmeli e Azeroual, 2009; Surroca et al., 2010; Martín-de-Castro et al., 2011; Kemmerer et al., 2012; Musiolik
Cinzia Dessì

Michela Floris

Il ruolo delle risorse Intangibili nella letteratura manageriale. Principali linee di ricerca e analisi future 


\section{sinergie}

Vol. 35, N. 103, 2017

et al., 2012; Poisson-de Haro e Montpetit, 2012; Silver Coley et al., 2012; Kianto et al., 2013; Kuivalainen et al., 2013; Norman et al., 2013; Soetanto e Jack, 2013).

(b.2) Analizzare la relazione tra RI e performance (Aragón-Sánchez e Sánchez-Marín, 2005; X Jiang, et al., 2011; Díaz-Fernández et al., 2015), anche in situazioni di incertezza (Hmieleski et al., 2015). Si noti che in quasi la totalità degli articoli gli autori riconoscono il ruolo delle RI nella realizzazione di performance superiori.

(b.3) Analizzare l'interazione tra crescita e vantaggio competitivo (Barney, 1991; Rangone, 1999; Haanes e Fjeldstad, 2000; Čater e Čater, 2009; Anderson e Eshima, 2013). Anche in questo caso, come nel precedente, questi articoli riconoscono la relazione tra RI e vantaggio competitivo.

(b.4) Individuare strumenti di valutazione e misurazione delle RI (Bontis, Dragonetti et al., 1999; Cruz-Ros, 2009; García-Merino et al., 2010; Molloy et al., 2011).

(b.5) Analizzare specifici assets (Rao, 1994; Dorsch e Carlson, 1996; McMillan e Deeds, 1998; Ambrosini e Bowman, 2001; Styhre, 2004; Windsperger, 2004; Kor e Leblebici, 2005; Salman e Saives, 2005; Branco e Rodrigues, 2006; Mathwick et al., 2008; Moustaghfir, 2009; Obloj e Capron, 2011; Arevalo et al., 2013; Atherton, 2013; Fernández-Olmos e Díez-Vial, 2013; Méndez-Durón, 2013; Gu e Lu, 2014; Xu Jiang et al., 2015) e le modalità attraverso le quali gli stessi possano essere contabilizzati (Catalfo e Wulf, 2016).

(b.6) Studiare il ruolo delle RI nella definizione della strategia di crescita, creazione di valore e innovazione d'impresa (Andersen e Kheam, 1998; Del Canto e Gonzalez, 1999; Camelo-Ordaz et al., 2003; Pike, Roos et al., 2005; Nisar et al., 2007; Richey et al., 2008; Gubbi et al., 2010; Padgett e Galan, 2010; Gómez e Vargas, 2012; Elango e Pattnaik, 2013; Denicolai et al., 2014; Paradkar et al., 2015; Montresor e Vezzani, 2016).

\subsection{C) Analisi delle teorie di riferimento e della metodologia utilizzata}

La teoria maggiormente utilizzata è la Resource Based View - RBV (Barney, 1991), seguita dalla Knowledge Based View - KBV - (Grant, 1996; Nonaka et al., 2000), dalla Dynamic Capabilities - DC - (Teece et al., 1997) e dalla Human Capital Theory - HCT - (Becker, 1994; Sweetland, 1996).

La RBV considera l'impresa come un "contenitore" di risorse tangibili e intangibili (Penrose, 1959; Wernerfelt, 1984) che consentono alle imprese di differenziarsi rispetto alle concorrenti e generare nel tempo un vantaggio competitivo difendibile, il quale, in sostanza, dipende dal sistema di risorse strategiche di cui è dotata l'impresa. Le imprese, in tal senso, si differenziano in relazione alle risorse detenute le quali devono possedere un valore, essere rare, non perfettamente imitabili e non perfettamente sostituibili (Barney, 1991). Tali tipologie di risorse risiedono nelle capacità intellettuali, nella conoscenza e nelle competenze delle persone.

La KBV (Grant, 1996; Nonaka et al., 2000) è una teoria che trova il suo fondamento nella conoscenza che, adeguatamente gestita e diffusa attraverso processi di apprendimento organizzativo, crea valore per l'impresa. 
La teoria delle DC (Teece et al., 1997) analizza le capacità degli individui nel ridefinire e riqualificare conoscenze, competenze e risorse interne al fine di accrescere il proprio patrimonio conoscitivo (Vicari, 1995; Vicari e Verona, 2000) e adeguarlo ai mutamenti dell'ambiente in cui opera per mantenere un vantaggio competitivo duraturo.

La HCT (Sweetland, 1996) trova il suo fondamento nel capitale umano, considerato depositario della conoscenza necessaria per ottenere performance elevate. In tal senso, ogni organizzazione dovrebbe investire nelle persone, nella loro conoscenza e nella loro formazione.

Relativamente alla metodologia, gli articoli utilizzano metodi qualitativi (case study, multiple - case study, review e analisi teoriche) e metodi quantitativi (indagini campionarie, metodi di regressione statistici). L'oggetto di analisi è, per oltre l'80\% degli articoli, la piccolamedia impresa.

\subsection{D) Analisi del focus}

Nonostante la varietà di argomenti e di obiettivi degli articoli è possibile delineare quattro principali filoni di ricerca.

\subsubsection{Analisi delle RI e loro classificazione}

È il filone più numeroso, i cui articoli qualificano e classificano le RI, oppure analizzano specifici e ben definiti assets. La definizione maggiormente utilizzata è quella proposta da Hall (1993) già richiamata in precedenza. Kianto et al. (2013) definiscono le RI come quelle risorse rappresentate dalla conoscenza, dal capitale umano, strutturale, dalle reti di relazioni e dalla gestione delle stesse risorse. Per quanto concerne la classificazione delle RI, alcuni studiosi (Harvey e Lusch, 1995; CameloOrdaz et al., 2003) riprendono quella proposta da Hall (1992) distinguendo le RI in: "assets" e " competencies".

Carmeli (2004), basandosi sul contributo di Barney (1991), presenta le caratteristiche peculiari delle risorse affinché possano definirsi strategiche. Altri autori, invece, soffermano la propria attenzione sui connotati della non trasferibilità, ambiguità e non imitabilità (Norman et al., 2013).

Diefenbach (2006) distingue le risorse immateriali da quelle materiali in relazione a: immaterialità; rinnovabilità; dinamismo. Miller e Shamsie (1996) propongono una distinzione tra risorse "property-based" e "knowledge-based".

\subsubsection{Analisi di specifiche RI}

Le RI maggiormente prese in considerazione sono: la conoscenza (Ambrosini e Bowman, 2001; Styhre, 2004; Windsperger, 2004; Moustaghfir, 2009; Atherton, 2013; Naldi e Davidsson, 2014), e la reputazione (Rao, 1994; McMillan e Deeds, 1998; Branco e Rodrigues, 2006; Obloj e Capron, 2011; Arevalo et al., 2013; Fernández-Olmos e DíezVial, 2013; Méndez-Durón, 2013; Gu e Lu, 2014; Raithel e Schwaiger, 2015; Ramalho e Resende, 2016).

La conoscenza costituisce una risorsa peculiare di ciascuna impresa, difficile, se non impossibile da imitare e fondamentale per la creazione di
Cinzia Dessì

Michela Floris

Il ruolo delle risorse

Intangibili nella letteratura

manageriale. Principali linee

di ricerca e analisi future 
sinergie Vol. 35, N. 103, 2017

valore (Teece et al., 1997). La RBV identifica nella conoscenza un aspetto fondamentale della competitività di un'impresa (Barney, 1991). Hall (1993) considera la conoscenza come una RI molto difficile da imitare a causa delle sue caratteristiche. Atherton (2013) affronta i temi della conoscenza distinguendola in: comune, disponibile, persa, non trasferibile, individuale, locale e disponibile.

Styhre (2004) si sofferma sulla conoscenza tacita, definendola come quel qualcosa che sfugge alla misurazione e rappresentazione, ma che riveste un'importanza fondamentale per l'impresa.

La seconda tipologia di RI maggiormente analizzata è la reputazione, difficile da misurare e valutare, ma dalla quale deriva la legittimazione dall'ambiente in cui l'impresa opera (Méndez-Durón, 2013).

Questa particolare risorsa dipende dalle performance precedenti dell'impresa, la sua responsabilità sociale, investimenti, ecc. e consente di rafforzare il brand, incrementare la fiducia dei consumatori e pertanto ottenere maggiori profitti (Fernández-Olmos e Díez-Vial, 2013). Roberts e Dowling (2002) affermano che la reputazione è una risorsa strategica in grado di accrescere le performance finanziare nel tempo e può essere identificata come una entità socialmente costruita e data da processi di legittimazione (Rao, 1994). Obloj e Capron (2011) introducono il concetto di reputation gap, ossia il differenziale di reputazione tra un'impresa, detta focale e un suo diretto concorrente, ritenendo che il reputation gap offra all'impresa focale la possibilità di definire un prezzo maggiore (prezzo "premio") rispetto al proprio concorrente, dato proprio dalla sua reputazione superiore. Tuttavia, creare e sviluppare una buona reputazione, richiede tempo e investimenti duraturi (Branco e Rodrigues, 2006). Particolarmente interessante è anche il contributo di Srivastava et al. (1998) che definiscono le relazioni con i clienti e con i partner come "market-based assets"(Srivastava et al., 1998). Anche Navarro et al. (2014) affrontano la tematica delle relazioni quali elementi per la co-creazione di valore nel settore dei servizi, con specifico focus verso i clienti affetti da disabilità.

Infine, numerosi contributi evidenziano la relazione intercorrente tra le RI, la performance e il vantaggio competitivo (Barney, 1991; Andersen e Kheam, 1998; Rangone, 1999; Camelo-Ordaz et al., 2003; Aragón Sánchez e Sánchez Marín, 2005; Čater e Čater, 2009), anche in termini di manovre strategiche promosse dai manager (Li e Tang, 2010), di condivisione di risorse nelle alleanze strategiche (Xu Jiang et al., 2015; Paradkar et al., 2015) di strategie di internazionalizzazione (Boehe, 2016) e di acquisizioni di conoscenza "internazionale" per la crescita delle imprese (Naldi e Davidsson, 2014). In questo senso, Čater e Čater (2009) sostengono che tra i fattori interni il capitale umano, quello strutturale e il customer capital, rappresentano elementi chiave per il vantaggio competitivo e le performance. Altri autori (Lai et al., 2015) individuano in alcune risorse intangibili (gratitudine, benevolenza, brevetti, capitale umano) i fattori che influenzano principalmente le decisioni in tema di Ricerca e Sviluppo. Altri autori (Aragón-Sánchez e Sánchez-Marín, 2005; Nachum e Zaheer, 2005) identificano nello sviluppo tecnologico e nell'innovazione i principali drivers per la competitività di un'impresa. Van Weele et al. (2017) affrontano la tematica dell'acquisizione di RI per le start up attraverso l'incubatore e il ricorso ad una strategia assertiva. Un aspetto interessante è l'introduzione 
del concetto di "asimmetria" (Miller, 2003) nel senso di differenziale di capacità possedute, il quale determina il punto di forza o di debolezza di un'impresa rispetto alle concorrenti.

\subsubsection{Misurazione e gestione delle RI}

Partendo dal presupposto che la RBV attribuisce una profonda rilevanza alle RI (Barney, 2001), misurarle e valutarle non è semplice. Bontis et al. (1999) presentano una literature review dei quattro strumenti maggiormente adottati per la misurazione e gestione delle RI, ossia: Human Resource Accounting (HRA), Economic Value Added (EVA), Balanced Scorecard (BS), Intellectual capital (IC). Tali strumenti hanno numerosi limiti dovuti alla soggettività e mancanza di affidabilità derivanti dall'astrattezza delle RI. La Balanced Scorecard viene riconosciuta come uno strumento potenziale e importante, pur presentando dei limiti connessi ad una rigidità dello strumento e ad una sottostima del valore della conoscenza. In sintesi si tratta di strumenti potenzialmente utili, la cui scelta e utilizzo dipende dalla tipologia di impresa e attività. Anderson e Eshima (2013) hanno sviluppato un modello per la misurazione del vantaggio che trova fondamento nella percezione dei manager. Molloy et al. (2011) sostengono che a tal fine possono essere utilizzati due specifici approcci, economico e psicologico, con i relativi tools di riferimento e che per sopperire ai limiti individuali è necessaria un'integrazione dei due metodi. Cruz-Ros (2009) ripropone il modello di misurazione definito da Camisón (2004), che si basa sulla percezione dei manager di detenere particolari risorse rispetto ai propri competitor e viene incentrato su "Managerial Resources" e "Organisational Resources". Hejazi et al. (2016) suggeriscono la misurazione dell'impatto del capitale umano, intellettuale e strutturale sulle performance attraverso lo strumento "Tobin's Q" (misura delle performance basata sul mercato). Recentemente, Zakery e Afrazeh (2017) hanno proposto un algoritmo per analizzare l'Intellectual capital Management con particolare enfasi nei confronti delle strategie di crescita.

In definitiva, la maggior parte degli strumenti presentati si fonda sulla percezione interna dei managers nel riconoscere la presenza di particolari risorse rispetto ai propri competitors.

\subsubsection{RI e vantaggio competitivo}

In questo filone confluiscono $i$ contributi che analizzano le relazioni che intercorrono tra RI, vantaggio competitivo, performance, crescita e creazione di valore. In questo quadro, autori come Norman et al. (2013) sostengono che le RI rivestono un ruolo determinante rispetto a quelle tangibili, nel perseguimento di un vantaggio competitivo per tre differenti aspetti: a) gli assets non possono essere facilmente scambiati e pertanto devono essere prodotti internamente; b) la relazione tra RI e vantaggio competitivo è ambigua, difficilmente comprensibile e misurabile; c) i concorrenti non riescono a replicare le RI e perseguire il medesimo risultato. Anche Carmeli (2004) sostiene che le RI detengono un ruolo fondamentale nel perseguimento di un vantaggio competitivo sostenibile e a tal proposito presenta differenti contributi nei quali si asserisce che performance più elevate dipendono dalla capacità innovativa di un'impresa 
sinergie Vol. 35, N. 103, 2017

e quindi conseguentemente dalla sua conoscenza. Le RI consentono di raggiungere un vantaggio competitivo in quanto non sono consumabili e possono essere utilizzate simultaneamente in differenti contesti. Hall (1992) ritiene che tra le RI ve ne siano alcune principali, quali il capitale umano, la reputazione e la cultura organizzativa, considerate le tre risorse più preziose in termini di contributo alla creazione di un vantaggio competitivo sostenibile. Un altro importante contributo proviene da Fernández et al. (2000), i quali evidenziano l'importanza delle RI per il conseguimento di un vantaggio competitivo poiché trattasi di risorse strettamente collegate alla conoscenza che non tendono a consumarsi con il decorrere del tempo ma anzi, accrescono il proprio valore con l'uso, mediante ripetizione e sperimentazione, fatta eccezione per il capitale tecnologico. Inoltre, le RI possono essere considerate fattori cruciali per lo sviluppo della crescita e per la creazione di sinergie (Fernández et al., 2000). Anderson e Eshima (2013) sostengono che le imprese che detengono un vantaggio derivante dalla presenza di RI, dovrebbero contestualmente detenere o manifestare un rafforzamento dell'orientamento imprenditoriale e della crescita. La presenza di "turmoil", ossia di un cambiamento significativo graduale o improvviso, derivante dall'ambiente interno o esterno dell'organizzazione, impone un'adeguata combinazione delle risorse umane, organizzative e fisiche al fine di incidere positivamente sulle risorse reputazionali e finanziarie (Poisson-de Haro e Montpetit, 2012).

X Jiang et al. (2011) evidenziano l'esistenza di una forte relazione positiva tra RI e innovazione e RI e performance superiori; tuttavia affermano che sebbene le RI rappresentino una fonte di vantaggio competitivo, nel momento in cui la protezione della proprietà intellettuale è debole, allora queste possono influenzare negativamente le performance finanziarie. Hervas-Oliver et al. (2016) indagano sui benefici che l'innovazione tecnologica può trarre dall'introduzione di innovazioni organizzative a livello manageriale. I lavori di Čater e Čater (2009) mettono in mostra che tra le RI viene attribuita particolare importanza a tre differenti elementi, quali human capital, structural capital e customer capital. Il primo è rappresentato dalle capacità individuali, abilità, esperienza, conoscenza degli individui; il secondo è dato dalla personificazione, responsabilizzazione e presenza di infrastrutture di supporto al capitale umano; infine il customer capital è costituito dalla conoscenza acquisita dalle relazioni con i clienti. Altri autori pongono laccento sul fatto che il processo mediante il quale l'impresa genera valore non è ancora chiaro, pertanto, identificando le RI come competenze e relazioni, non è possibile stabilire con certezza quando questi due elementi siano più critici nel processo di creazione (Haanes e Fjeldstad, 2000). Nonostante i risultati presentati mettano in evidenza il ruolo centrale detenuto dalle RI nel perseguimento di performance più elevate, è doveroso presentare anche il contributo di Elango e Pattnaik (2013), i quali sottolineano l'importanza dello studio condotto da Villalonga (2004) che mostra come le RI possono anche bloccare l'impresa e comportare svantaggi persistenti a causa di "core rigidities" nellorganizzazione. 


\section{Discussione dei risultati}

Lo studio e l'analisi critica comparata dei 95 articoli evidenziano l'esistenza di un framework teorico piuttosto frammentario che necessita di ulteriori sforzi per una più compiuta e operativa analisi del fenomeno. L'unico aspetto sul quale vi è quasi unanimità tra gli studiosi è l'esistenza di una relazione tra RI, performance, vantaggio competitivo e crescita aziendale. Tale relazione è positiva nel momento in cui le imprese individuano e isolano le RI che ne costituiscono l'essenza e le differenziano dalle concorrenti.

Sulla base di questi assunti, si intende proporre un modello interpretativo (Fig. 1) che consideri congiuntamente gli studi già pubblicati e identifichi nuovi scenari ancora inesplorati, al fine di arricchire l'attuale letteratura sul tema e di fornire supporti operativi ai practitioner.

Fig. 1: Un modello interpretativo

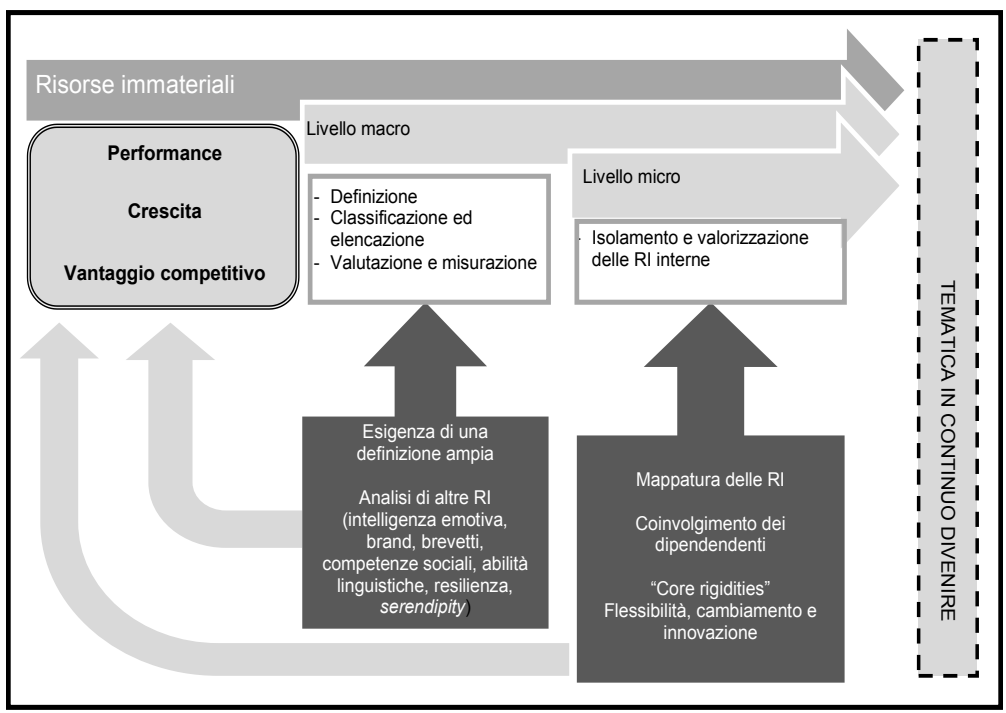

Fonte: nostra elaborazione

Il modello si basa sulla concezione unanime che le RI influenzano la performance delle imprese, determinandone la crescita e concorrendo alla creazione di un duraturo e difendibile vantaggio competitivo.

Il livello di analisi è duplice: da un lato, si osservano le variabili che influenzano il livello "macro", ossia la generalità delle imprese e, dall'altro lato, il livello "micro", ossia ciò che si ritiene fondamentale approfondire all'interno di ogni singola impresa. È rilevante sottolineare che i livelli sono solo astrattamente distinti, in quanto nella realtà le dinamiche macro e micro tendono a sovrapporsi e ad autoalimentarsi. Tuttavia, la finalità del modello è quella di rappresentare la realtà in un modo meno complesso, per poter inquadrare il fenomeno e analizzarlo ad un livello di astrazione superiore.

A livello "macro", le criticità emerse dall'analisi sono riconducibili alle problematiche derivanti dall'inesistenza di una definizione unanime
Cinzia Dessì

Michela Floris

Il ruolo delle risorse

Intangibili nella letteratura

manageriale. Principali linee

di ricerca e analisi future 
sinergie Vol. 35, N. 103, 2017

che consenta di racchiudere un fenomeno multidimensionale in un unico ampio costrutto. Conseguentemente, l'assenza di una compiuta classificazione delle RI rischia di creare effetti negativi sullo sforzo di "isolamento" e sviluppo delle RI critiche che rappresentano la principale fonte di vantaggio competitivo delle imprese. Inoltre, l'assenza di un inquadramento condiviso e di unelencazione esaustiva delle RI genera difficoltà nella creazione di congrui e validi sistemi di misurazione e valutazione che, allo stato attuale, esistono, ma che necessitano di ulteriori implementazioni e approfondimenti. Ciò non significa, ovviamente, che gli studiosi abbiano tralasciato la tematica. Piuttosto, dall'analisi è emersa una propensione nei confronti di alcune specifiche RI (capitale umano, conoscenza, reputazione, capitale intellettuale, cultura organizzativa).

Relativamente al livello "micro", quello cioè maggiormente focalizzato sulla singola impresa, il modello si basa sullesigenza di riconoscere, isolare e valorizzare specifiche RI interne per poter perseguire elevate performance. A tal proposito sarebbe interessante concepire uno strumento atto a mappare le RI interne e valutarne il loro impatto nelle performance. Il coinvolgimento dei dipendenti si ritiene fondamentale nell'identificazione e conseguente potenziamento delle RI, con particolare attenzione a ciò che emerge dagli studi di Villalonga (2004) in tema di "core rigidities" e dei potenziali svantaggi che potrebbero derivare da esse. Per questo motivo, il modello considera tre importanti variabili: flessibilità, cambiamento e innovazione (Richey et al., 2008; Gómez e Vargas, 2012), al fine di evitare l'insorgere di correlazioni negative tra RI e performance.

Gli assunti sui quali poggia il modello, derivanti dallanalisi della letteratura, descrivono un quadro teorico in continuo divenire che necessita di ulteriori sforzi.

\section{Alcuni spunti per future ricerche}

Come già evidenziato, il modello interpretativo proposto presenta unanalisi dello stato dell'arte e si propone di analizzare la tematica delle RI da due punti di vista: macro e micro. Da questo quadro emergono alcuni interessanti spunti per ricerche future che possono essere ripartiti in tre ideali gruppi, in relazione a:

1. RI analizzate;

2. campo di osservazione;

3. integrazione con nuovi fenomeni emergenti.

Per quanto riguarda il primo gruppo, dallanalisi del contenuto dei 95 articoli è emerso che le RI maggiormente analizzate sono: capitale umano, conoscenza, reputazione, capitale intellettuale, cultura organizzativa. Tuttavia, in relazione ai cambiamenti che lo scenario socio-economico sta attualmente vivendo, alcune RI ugualmente strategiche appaiono del tutto trascurate o citate solo marginalmente (intelligenza emotiva, creatività, brand, brevetti, competenze sociali, abilità linguistiche, resilienza e serendipity). Sarebbe interessante analizzare queste RI, il contesto nel quale si manifestano, le modalità di emersione e trasferimento e la valutazione dell'impatto sulle performance, in particolare nei mercati internazionali, laddove è determinante sviluppare abilità e competenze sociali e comunicative per la creazione di alleanze strategiche. Degno di 
considerazione è anche l'approfondimento di specifiche RI atte a migliorare le relazioni e l'accessibilità a prodotti e servizi a soggetti diversamente abili (Navarro et al., 2014).

Relativamente al secondo gruppo, la prevalenza degli studi non presenta unanalisi accurata delle imprese osservate e del contesto in cui le stesse operano. Sarebbe particolarmente interessante approfondire lo studio delle RI nelle PMI, nelle imprese di medie dimensioni e in quelle più grandi e strutturate. Probabilmente le RI proprie di ciascuna categoria di impresa differiscono dalle altre e ciò potrebbe contribuire al dibattito in tema di asimmetrie di potenziale interno (Miller, 2003). Inoltre, si ritiene rilevante anche un'analisi del contesto nel quale le imprese sono radicate (fattore milieu), in particolare per le PMI che hanno una fitta rete di relazioni con il territorio di riferimento e dal quale traggono la prevalenza delle proprie risorse per poter sopravvivere, comprese quelle intangibili tra le quali emergono la reputazione, la popolarità, la fiducia, la notorietà e l'apprezzamento del territorio. In questo senso si contribuirebbe al dibattito esistente in tema di "Societing Reloaded" (Arvidsson e Giordano, 2013) e di "Società Interconnessa" (Dominici, 2014).

Infine, relativamente al terzo gruppo, il trend della letteratura più recente parrebbe suggerire l'integrazione dell'analisi delle RI con i fenomeni di internazionalizzazione delle imprese e di nascita di nuove iniziative imprenditoriali innovative (start up), soprattutto per quanto concerne le risorse relazionali e il capitale intellettuale. A questo andrebbe combinato anche un ulteriore aspetto dettato dalle nuove tendenze del mercato in tema di green economy e di flessibilità nei fenomeni innovativi. In questo senso, future ricerche potrebbero, da un lato, contribuire alla green economy theory (Rifkin, 2011) e, dall'altro, approfondendo le problematiche legate alle core rigidities (Villalonga, 2004) potrebbero contribuire al dibattito in tema di path dependence (Arthur, 1994; David, 1994; 2001; Donnelly, 2009).

\section{Conclusioni}

La tematica delle risorse intangibili è rilevante per il mondo accademico e per la realtà delle imprese.

I risultati della presente ricerca hanno contribuito al dibattito sul tema inquadrando il fenomeno e sistematizzando i contributi scientifici che lo hanno delineato. La review della letteratura ha infatti, da un lato, presentato il quadro dei principali studi pubblicati dal 1990 al mese di aprile 2017, individuando i principali filoni sui quali gli studiosi si sono soffermati e, dall'altro lato, proposto un modello interpretativo della tematica.

Loriginalità della ricerca risiede principalmente su tre elementi: a. spazio temporale ampio che abbraccia diverse fasi della ricerca scientifica e consente di analizzare la tematica partendo da contributi fondamentali e arricchendola di pubblicazioni più recenti; b. utilizzo della locuzione "Intangibles resources" come parola chiave, senza limitare la ricerca all'analisi di specifiche e ben definite risorse immateriali; c. analisi dei contributi scientifici riconducibili in senso lato al Management e al Business, diversamente da altre review della letteratura che si sono focalizzate su Finance, Accounting e altre aree.
Cinzia Dessì
Michela Floris

Il ruolo delle risorse

Intangibili nella letteratura

manageriale. Principali linee

di ricerca e analisi future 
sinergie Vol. 35, N. 103, 2017

Le principali limitazioni sono rinvenibili nel database scelto per l'analisi. Tuttavia, tale problematica è stata parzialmente risolta combinando l'uso di due banche dati che hanno consentito di ampliare il numero e la varietà di articoli da analizzare.

Alla luce della rilevanza della tematica, anche in virtù del particolare momento storico che le imprese stanno vivendo, caratterizzato da risorse limitate e crisi generalizzata, le implicazioni del presente studio sono sia di natura accademica sia di natura pratica.

Per quanto concerne le prime, la presente ricerca contribuisce a incrementare la letteratura manageriale sul tema delle RI offrendo un quadro d'insieme dei contributi scientifici pubblicati su due dei principali database utilizzati in campo scientifico. L'individuazione dei principali filoni di ricerca ha consentito di individuare potenziali nuove linee future, che dovrebbero andare a colmare un'esigenza di analisi e approfondimento motivata dalla rilevanza che le RI rivestono per tutte le categorie di imprese. Il modello interpretativo, in particolare, analizzando la tematica da un punto di vista "macro" e "micro", arricchisce la letteratura sistematizzando i contributi esistenti, individuando alcune esigenze e proponendo tre nuovi percorsi di studio che evidenziano lesistenza di un framework teorico in continuo divenire.

Relativamente alle implicazioni pratiche, è importante sottolineare come le imprese siano interessate internamente ed esternamente a misurare e gestire positivamente le proprie RI. Il mondo finanziario, infatti, ruota intorno al concetto di RI e nellattuale contesto storico, connotato fortemente da limitati capitali, i potenziali investitori sono interessati a conoscere informazioni aggiuntive che un bilancio desercizio non è in grado, da solo, di fornire. Conoscere e presentare ai potenziali investitori informazioni sulle proprie RI può creare vantaggi nel mercato finanziario e offrire ulteriori garanzie. Per questo motivo manager e imprenditori sono chiamati a individuare le RI che caratterizzano le proprie imprese, al fine di conoscere gli elementi verso i quali investire risorse. Unanalisi compiuta delle RI proprie di ciascuna impresa, infatti, potrebbe supportare la definizione delle strategie da implementare e consentirebbe di avere probabilità superiori nel reperimento delle fonti di finanziamento, nonché di creare un'immagine esterna conforme alle caratteristiche reali dell'impresa. Le RI potrebbero essere, infatti, fattori di correzione dei modelli di rating. Ciò deriva dal fatto che si tratta di elementi rari e inimitabili che consentono all'impresa di creare un vantaggio competitivo duraturo e sostenibile. Indubbiamente la sostenibilità delle iniziative imprenditoriali è una caratteristica dell'impresa fondamentale per l'accesso al credito. Tuttavia, poiché le RI non sono facilmente misurabili e la loro valutazione in bilancio non è semplice proprio alla luce della loro "intangibilità", le imprese sono chiamate a fornire informazioni aggiuntive e a delineare in modo compiuto quali sono le proprie RI. I dettami di Basilea III hanno attribuito facoltà alle banche di individuare le modalità di combinazione tra elementi hard (variabili quantitative e di natura finanziaria) e soft (variabili qualitative e di natura non finanziaria) e poiché le imprese, in particolare quelle di medie e piccole dimensioni, non sempre possiedono adeguate informazioni hard, l’approccio più utile nella valutazione del rischio del credito potrebbe essere quello soft, all'interno del quale le RI giocano un ruolo importante (Quintiliani, 2016). 
AMBROSINI V., BOWMAN C. (2001), “Tacit knowledge: Some suggestions for operationalization", Journal of Management Studies, vol. 38, n. 6, pp. 811di ricerca e analisi future 829.

ANDERSEN O., KHEAM L.S. (1998), "Resource-based theory and international growth strategies: an exploratory study", International Business Review, vol. 7, n. 2, pp. 163-184.

ANDERSON B.S., ESHIMA Y. (2013), "The influence of firm age and intangible resources on the relationship between entrepreneurial orientation and firm growth among Japanese SMEs", Journal of Business Venturing, vol. 28, n. 3, pp. 413-429.

ARAGÓN-SÁNCHEZ A., SÁNCHEZ-MARÍN G. (2005), "Strategic orientation, management characteristics, and performance: A study of Spanish SMEs", Journal of Small Business Management, vol. 43, n. 3, pp. 287-308.

AREVALO J.A., ARAVIND D., AYUSO S., ROCA M. (2013), “The Global Compact: An analysis of the motivations of adoption in the Spanish context", Business Ethics: A European Review, vol. 22, n. 1, pp. 1-15.

ARTHUR W.B. (1994), Increasing returns and path dependence in the economy, University of Michigan Press, Ann Arbor, MI.

ARVIDSSON A., GIORDANO A. (2013), Societing Reloaded: pubblici produttivi e innovazione sociale, EGEA, Milano.

ATHERTON A. (2013), "Organisational 'know-where'and 'know-when': reframing configurations and distributions of knowledge in organisations", Knowledge Management Research and Practice, vol. 11, n. 4, pp. 410-421.

BARNEY J.B. (1991), "Firm resources and sustained competitive advantage", Journal of Management, vol. 17, n. 1, pp. 99-120.

BARNEY J.B. (1997), Gaining and sustaining competitive advantage, AddisonWesley Reading, MA Massachusetts.

BARNEY J.B. (2001), "Is the resource-based "view" a useful perspective for strategic management research? Yes", Academy of Management Review, vol. 26, n. 1, pp. 41-56.

BAUMEISTER R.F., LEARY M.R. (1997), "Writing narrative literature reviews", Review of General Psychology, vol. 1, n. 3, pp. 311-320.

BECKER G.S. (1994), Human Capital: A Theoretical and Empirical Analysis, with Special Reference to Education, The University Chicago Press, Chigago.

BOEHE D.M. (2016), "The internationalization of service firms from emerging economies: An internalization perspective", Long Range Planning, vol. 49, n. 5, pp. 559-569.

BONTIS N., BART C., WAKEFIELD P., KRISTANDL G., BONTIS N. (2007), "Constructing a definition for intangibles using the Resource Based View of the firm", Management Decision, vol. 45, n. 9, pp. 1510-1524.

BONTIS N., DRAGONETTI N.C., JACOBSEN K., ROOS G. (1999), “The knowledge toolbox: A review of the tools available to measure and manage intangible resources", European Management Journal, vol. 17, n. 4, pp. 391402.

BRANCO M.C., RODRIGUES L.L. (2006), "Corporate social responsibility and resource-based perspectives", Journal of Business Ethics, vol. 69, n. 2, pp. 111-132.

BRONDONI S.M. (2002), "Comunicazione, performance e sistema delle risorse immateriali d'impresa”, Sinergie, n. 59, pp. 41-52. 


\section{sinergie} Vol. 35, N. 103, 2017
CAMELO-ORDAZ C., MARTÍN-ALCÁZAR F., VALLE-CABRERA R. (2003), "Intangible resources and strategic orientation of companies: An analysis in the Spanish context", Journal of Business Research, vol. 56, n. 2, pp. 95103.

CAMISÓN C. (2004), "Shared, competitive, and comparative advantages: a competence-based view of industrial-district competitiveness", Environment and Planning A, vol. 36, n. 12, pp. 2227-2256.

CAMISÓN C., VILLAR A. (2009), "Capabilities and propensity for cooperative internationalization", International Marketing Review, vol. 26, n. 2, pp. 124150.

CAÑIBANO L., GARCIA-AYUSO M., SANCHEZ P. (2000), "Accounting for intangibles: a literature review", Journal of Accounting Literature, vol. 19, pp. 102-130.

CARMELI A. (2004), "Assessing core intangible resources", European Management Journal, vol. 22, n. 1, pp. 110-122.

CARMELI A., AZEROUAL B. (2009), "How Relational Capital and Knowledge Combination Capability Enhance the Performance of Work Units in a High Technology Industry", Strategic Entrepreneurship Journal, vol. 3, n. 1, pp. 85-103.

CATALFO P., WULF I. (2016), "Intangibles disclosure in Management Commentary regulation in Germany and Italy", Journal of Intellectual Capital, vol. 17, n. 1, pp. 103-119.

ČATER T., ČATER B. (2009), “(In)tangible resources as antecedents of a company’s competitive advantage and performance", Journal for East European Management Studies, vol. 14, n. 2, pp. 186-209.

CEPEDA G., VERA D. (2007), "Dynamic Capabilities and operational capabilities: A knowledge management perspective", Journal of Business Research, vol. 60 , n. 5, pp. 426-437.

COOPER, H. (1998), Synthesizing Research: A Guide for Literature reviews, Thousand Oaks, CA., Sage Publications.

CROOK T.R., KETCHEN D.J., COMBS J.G., TODD S.Y. (2008), “Strategic resources and performance: a meta-analysis", Strategic Management Journal, vol. 29, n. 11, pp. 1141-1154.

CRUZ-ROS S. (2009), "Multi-item models for evaluating managerial and organizational resources in service firms", Service Business, vol. 3, n. 3, pp. 229-257.

D’AVENI R.A., GUNTHER R.E. (1995), Hypercompetitive rivalries: Competing in highly dynamic environments, Free Press, New York

DAVID P.A. (1994), "Why are institutions the 'carriers of history'?: Path dependence and the evolution of conventions, organizations and institutions", Structural change and economic dynamics, vol. 5, n. 2, pp. 205-220.

DAVID P.A. (2001), "Path dependence, its critics and the quest for "historical economics", in Garrouste P., Ioannides S. (Eds.), Evolution and path dependence in economic ideas: Past and present, Edward Elgar, Cheltenham, UK.

DEL CANTO J.G., GONZALEZ I.S. (1999), "A resource-based analysis of the factors determining a firm's R\&D activities", Research Policy, vol. 28, n. 8, pp. 891-905.

DENICOLAI S., RAMIREZ M., TIDD J. (2014), "Creating and capturing value from external knowledge: the moderating role of knowledge intensity", R\&D Management, vol. 44, n. 3, pp. 248-264. 
DÍAZ-FERNÁNDEZ M.C., GONZÁLEZ-RODRÍGUEZ M.R., SIMONETTI B. (2015), "Top management team's intellectual capital and firm performance", European Management Journal, vol. 33, n. 5, pp. 322-331.

DIEFENBACH, T. (2006), "Intangible resources: a categorial system of knowledge and other intangible assets", Journal of Intellectual capital, vol. 7, n. 3, pp. 406-420.

DIXON-WOODS M., BONAS S., BOOTH A., JONES D.R., MILLER T., SUTTON A.J., SHAW R.L., SMITH J.A., YOUNG B. (2006), "How can systematic review s incorporate qualitative research? A critical perspective", Qualitative Research, vol. 6, n. 1, pp. 27-44.

DOMINICI P. (2014), Dentro la società interconnessa, Franco Angeli, Milano.

DONNELLY P. (2009), "Focusing on Process and History: Path dependence", in Hogan J., Dolan P., Donnelly P. (Eds.), Approaches to Qualitative Research: Theory and Its Practical Application, O.T. Press, Cork, IE.

DORSCH M.J., CARLSON L. (1996), "A transaction approach to understanding and managing customer equity", Journal of Business Research, vol. 35, n. 3, pp. 253-264.

ELANGO B., PATTNAIK C. (2013), "Response strategies of local firms to import competition in emerging markets", Journal of Business Research, vol. 66, n. 12, pp. 2460-2465.

FERNÁNDEZ-OLMOS M., DÍEZ-VIAL I. (2013), "Effect of firm's resources on international diversification: An application in the Iberian Ham industry", European Management Journal, vol. 31, n. 2, pp. 196-208.

FERNÁNDEZ E., MONTES J.M., VÁZQUEZ C.J. (2000), "Typology and strategic analysis of intangible resources: a resource-based approach", Technovation, vol. 20, n. 2, pp. 81-92.

GALBREATH, J. (2005), "Which resources matter the most to firm success? An exploratory study of resource-based theory", Technovation, vol. 25, n. 9, pp. 979-987.

GARCÍA-MERINO J.D., ARREGUI-AYASTUY G., RODRÍGUEZ-CASTELLANOS A., GARCÍA-ZAMBRANO L. (2010), "The intangibles' mindset of CFOs' and corporate performance", Knowledge Management Research and Practice, vol. 8, n. 4, pp. 340-350.

GARDNER T.M. (2005), "Interfirm competition for human resources: Evidence from the software industry", Academy of Management Journal, vol. 48, $\mathrm{n}$. 2, pp. 237-256.

GÓMEZ J., VARGAS P. (2012), "Intangible resources and technology adoption in manufacturing firms", Research Policy, vol. 41, n. 9, pp. 1607-1619.

GOTTFREDSON M., ASPINALL K. (2005), "Innovation versus complexity: What is too much of a good thing?", Harvard Business Review, vol. 83, n. 11, pp. $62-71$.

GRANT R.M. (1996), "Prospering in dynamically-competitive environments: Organizational capability as knowledge integration", Organization Science, vol. 7, n. 4, pp. 375-387.

GRAY D., ROOS G., RASTAS T. (2004), "What intangible resources do companies value, measure, and report? A synthesis of UK and Finnish research", International Journal of Learning and Intellectual capital, vol. 1, n. 3, pp. 242-261.

GU Q., LU X. (2014), "Unraveling the mechanisms of reputation and alliance formation: A study of venture capital syndication in China", Strategic Management Journal, vol. 35, n. 5, pp. 739-750. 
GUBBI S.R., AULAKH P.S., RAY S., SARKAR M., CHITTOOR R. (2010), “Do international acquisitions by emerging-economy firms create shareholder value? The case of Indian firms", Journal of International Business Studies, vol. 41, n. 3, pp. 397-418.

HAANES K., FJELDSTAD Ø. (2000), "Linking intangible resources and competition", European Management Journal, vol. 18, n. 1, pp. 52-62.

HALL R. (1992), "The strategic analysis of intangible resources", Strategic Management Journal, vol. 13, n. 2, pp. 135-144.

HALL R. (1993), "A framework linking intangible resources and capabiliites to sustainable competitive advantage", Strategic Management Journal, vol. 14, n. 8, pp. 607-618.

HARRIS J. (2005), "Emerging third world powers: China, India and Brazil”, Race and Class, vol. 46, n. 3, pp. 7-27.

HARVEY M.G., LUSCH R.F. (1995), "Expanding the nature and scope of due diligence", Journal of Business Venturing, vol. 10, n. 1, pp. 5-21.

HAYTON, J. C. (2005), "Competing in the new economy: the effect of intellectual capital on corporate entrepreneurship in high-technology new ventures", R\&D Management, vol. 35, n. 2, pp. 137-155.

HEJAZI R., GHANBARI M., ALIPOUR M. (2016), "Intellectual, Human and Structural Capital Effects on Firm Performance as Measured by Tobin's Q", Knowledge and Process Management, vol. 23, n. 4, pp. 259-273.

HERVAS-OLIVER J.L., RIPOLL-SEMPERE F., MOLL C.B. (2016), "Does management innovation pay-off in SMEs? Empirical evidence for Spanish SMEs", Small Business Economics, vol. 47, n. 2, pp. 507-533.

HEWITT-DUNDAS N. (2006), "Resource and capability constraints to innovation in small and large plants", Small Business Economics, vol. 26, n. 3, pp. 257 277.

HMIELESKI K.M., CARR J.C., BARON R.A. (2015), "Integrating discovery and creation perspectives of entrepreneurial action: the relative roles of founding CEO human capital, social capital, and psychological capital in contexts of risk versus uncertainty", Strategic Entrepreneurship Journal, vol. 9, n. 4, pp. 289-312.

JIANG C.X., YANG Q., LI S., WANG Y. (2011), “The moderating effect of foreign direct investment intensity on local firms' intangible resources investment and performance implications: A case from China", Journal of International Management, vol. 17, n. 4, pp. 291-302.

JIANG X., JIANG F., CAI X., LIU H. (2015), "How does trust affect alliance performance? The mediating role of resource sharing", Industrial Marketing Management, vol. 45, pp. 128-138.

JONES R.W., KIERZKOWSKI H. (2003), “A Framework For Fragmentation”, in Fragmentation: new production patterns in the world economy, Arndt K. S.W., Oxford H., Oxford University Press.

KAUFMANN L., SCHNEIDER Y. (2004), "Intangibles: a synthesis of current research", Journal of Intellectual capital, vol. 5, n. 3, pp. 366-388.

KEMMERER B., WALTER J., KELLERMANNS F.W., NARAYANAN V. (2012), "A judgment-analysis perspective on entrepreneurs' resource evaluations", Journal of Business Research, vol. 65, n. 8, pp. 1102-1108.

KIANTO A., ANDREEVA T., PAVLOV Y. (2013), "The impact of intellectual capital management on company competitiveness and financial performance", Knowledge Management Research and Practice, vol. 11, n. 2, pp. 112-122. 
KOR Y.Y., LEBLEBICI H. (2005), "How do interdependencies among humancapital deployment, development, and diversification strategies affect firms' financial performance?", Strategic Management Journal, vol. 26, n. 10, pp. 967-985.

KUIVALAINEN O., SUNDQVIST S., SAARENKETO S., MCNAUGHTON R.B., D'ANGELO A., MAJOCCHI A., ZUCCHELLA A., BUCK T. (2013), "Geographical pathways for SME internationalization: insights from an Italian sample", International Marketing Review, vol. 30, n. 2, pp. 80-105.

LAI Y.L., LIN F.J., LIN Y.H. (2015), "Factors affecting firm's R\&D investment decisions", Journal of Business Research, vol. 68, n. 4, pp. 840-844.

LI J.T., TANG Y. (2010), "CEO hubris and firm risk taking in China: The moderating role of managerial discretion", Academy of Management Journal, vol. 53, n. 1, pp. 45-68.

MACINNIS D.J. (2011), "A framework for conceptual contributions in marketing", Journal of Marketing, vol. 75, n. 4, pp. 136-154.

MARCUS A.A., ANDERSON M.H. (2006), "A General Dynamic Capability: Does it Propagate Business and Social Competencies in the Retail Food Industry?", Journal of Management Studies, vol. 43, n. 1, pp. 19-46.

MARTÍN-DE-CASTRO G., DELGADO-VERDE M., LÓPEZ-SÁEZ P., NAVASLÓPEZ J.E. (2011), “Towards 'an intellectual capital-based view of the firm': origins and nature", Journal of Business Ethics, vol. 98, n. 4, pp. 649662.

MATHWICK C., WIERTZ C., DE RUYTER K. (2008), "Social capital production in a virtual P3 community", Journal of Consumer Research, vol. 34, n. 6, pp. 832-849.

MCMILLAN G., DEEDS L. (1998), "The role of reputation in the recruitment of scientists”, ReD Management, vol. 28, n. 4, pp. 299-304.

MEHO L.I., YANG K. (2007), "Impact of data sources on citation counts and rankings of LIS faculty: Web of Science versus Scopus and Google Scholar", Journal of the American Society for Information Science and Technology, vol. 58 , n. 13, pp. 2105-2125.

MELVILLE N., KRAEMER K., GURBAXANI V. (2004), "Review : Information technology and organizational performance: An integrative model of IT business value", MIS quarterly, vol. 28, n. 2, pp. 283-322.

MÉNDEZ-DURÓN R. (2013), "Do the allocation and quality of intellectual assets affect the reputation of open source software projects?", Information and Management, vol. 50, n. 7, pp. 357-368.

MILLER D. (2003), "An asymmetry-based view of advantage: towards an attainable sustainability", Strategic Management Journal, vol. 24, n. 10, pp. 961-976.

MILLER D., SHAMSIE J. (1996), "The resource-based view of the firm in two environments: The Hollywood film studios from 1936 to 1965", Academy of management journal, vol. 39, n. 3, pp. 519-543.

MOLLOY J.C., CHADWICK C., PLOYHART R.E., GOLDEN S.J. (2011), "Making Intangibles "Tangible" in Tests of Resource-Based Theory A Multidisciplinary Construct Validation Approach", Journal of Management, vol. 37, n. 5, pp. 1496-1518.

MONTRESOR S., VEZZANI A. (2016), "Intangible investments and innovation propensity: Evidence from the Innobarometer 2013", Industry and Innovation, vol. 23, n. 4, pp. 331-352.

MOUSTAGHFIR K. (2009), "How knowledge assets lead to a sustainable competitive advantage: are organizational capabilities a missing link?", Knowledge Management Research and Practice, vol. 7, n. 4, pp. 339-355. 
MUSIOLIK J., MARKARD J., HEKKERT M. (2012), "Networks and network resources in technological innovation systems: Towards a conceptual framework for system building", Technological Forecasting and Social Change, vol. 79, n. 6, pp. 1032-1048.

NACHUM L., ZAHEER S. (2005), "The persistence of distance? The impact of technology on MNE motivations for foreign investment", Strategic Management Journal, vol. 26, n. 8, pp. 747-767.

NALDI L., DAVIDSSON P. (2014), "Entrepreneurial growth: The role of international knowledge acquisition as moderated by firm age", Journal of Business Venturing, vol. 29, n. 5, pp. 687-703.

NAVARRO S., ANDREU L., CERVERA A. (2014), "Value co-creation among hotels and disabled customers: An exploratory study", Journal of Business Research, vol. 67, n. 5, pp. 813-818.

NISAR T., MARTIN R., MAY SEITANIDI M. (2007), "Intangible economy: How can investors deliver change in businesses? Lessons from nonprofitbusiness partnerships”, Management Decision, vol. 45, n. 5, pp. 853-865.

NONAKA I., TOYAMA R., KONNO N. (2000), "SECI, Ba and leadership: a unified model of dynamic knowledge creation", Long Range Planning, vol. 33, n. 1, pp. 5-34.

NORMAN P.M., BUTLER F.C., RANFT A.L. (2013), "Resources Matter Examining the Effects of Resources on the State of Firms Following Downsizing", Journal of Management, vol. 39, n. 7, pp. 2009-2038.

OBLOJ T., CAPRON L. (2011), "Role of resource gap and value appropriation: Effect of reputation gap on price premium in online auctions", Strategic Management Journal, vol. 32, n. 4, pp. 447-456.

PADGETT R.C., GALAN J.I. (2010), "The effect of R\&D intensity on corporate social responsibility", Journal of Business Ethics, vol. 93, n. 3, pp. 407-418.

PARADKAR A., KNIGHT J., HANSEN P. (2015), "Innovation in start-ups: Ideas filling the void or ideas devoid of resources and capabilities?", Technovation, vol. 41-42, n. July-August, pp. 1-10.

PENROSE E. (1959), The theory of the growth of the firm, NY Wiley, New York.

PETTICREW M., ROBERTS H. (2008), Systematic review s in the social sciences: A practical guide, NY, John Wiley \& Sons, New York.

PIKE S., ROOS G., MARR B. (2005), "Strategic management of intangible assets and value drivers in R\&D organizations", R\&D Management, vol. 35, n. 2, pp. 111-124.

POISSON-DE HARO S., MONTPETIT D. (2012), "Surviving in Times of Turmoil: Adaptation of the Théâtre Les Deux Mondes Business Model", International Journal of Arts Management, vol. 14, n. 3, pp. 16-31.

PORTER M.E. (1985), Competitive advantage; creating and sustaining superior performance, The Free Press, New York, NY.

PORTER M.E. (1991), "Towards a dynamic theory of strategy", Strategic Management Journal, vol. 12, n. S2, pp. 95-117.

QUINTILIANI A. (2016), Internal rating systems e soft information: Il ruolo degli intangibili e del contesto territoriale nella valutazione del merito creditizio delle PMI, Franco Angeli, Milano.

RAITHEL S., SCHWAIGER M. (2015), "The effects of corporate reputation perceptions of the general public on shareholder value", Strategic Management Journal, vol. 36, n. 6, pp. 945-956.

RAMALHO W., RESENDE L.M. (2016), "Reputation in public transport service", Revista Gestão and Tecnologia, vol. 16, n. 2, pp. 218-237. 
RANGONE A. (1999), "A resource-based approach to strategy analysis in smallmedium sized enterprises", Small Business Economics, vol. 12, n. 3, pp. 233248.

RAO H. (1994), "The social construction of reputation: Certification contests, legitimation, and the survival of organizations in the American automobile industry: 1895-1912", Strategic Management Journal, vol. 15, n. S1, pp. 2944.

RICHEY R.G., TOKMAN M., SKINNER L.R. (2008), "Exploring collaborative technology utilization in retailer-supplier performance", Journal of Business Research, vol. 61, n. 8, pp. 842-849.

RIFKIN J. (2011), The third industrial revolution: how lateral power is transforming energy, the economy, and the world, Palgrave Macmillan, New York.

ROBERTS P.W., DOWLING G.R. (2002), "Corporate reputation and sustained superior financial performance”, Strategic Management Journal, vol. 23, n. 12, pp. 1077-1093.

RODRIGUEZ J.L., RODRÍGUEZ R.M.G. (2005), "Technology and export behaviour: A resource-based view approach”, International Business Review, vol. 14, n. 5, pp. 539-557.

RUBERA G., KIRCA A.H. (2012), "Firm innovativeness and its performance outcomes: A meta-analytic review and theoretical integration", Journal of Marketing, vol. 76, n. 3, pp. 130-147.

RUMRILL JR. P.D., FITZGERALD S.M. (2001), “Using narrative literature reviews to build a scientific knowledge base", Work, vol. 16, n. 2, pp. 165-170.

SALDAÑA J. (2012), The coding manual for qualitative researchers, Sage, London.

SALMAN N., SAIVES A.L. (2005), "Indirect networks: an intangible resource for biotechnology innovation”, R\&D Management, vol. 35, n. 2, pp. 203-215.

SILVER COLEY L., LINDEMANN E., WAGNER S.M. (2012), "Tangible and intangible resource inequity in customer-supplier relationships", Journal of Business and Industrial Marketing, vol. 27, n. 8, pp. 611-622.

SOETANTO D.P., JACK S.L. (2013), "Business incubators and the networks of technology-based firms", The Journal of Technology Transfer, vol. 38, n. 4, pp. 432-453.

SRIVASTAVA R.K., SHERVANI T.A., FAHEY L. (1998), "Market-based assets and shareholder value: A framework for analysis", Journal of Marketing, vol. 62, n. 1, pp. 2-18.

STYHRE A. (2004), "Rethinking Knowledge: A Bergsonian Critique of the Notion of Tacit Knowledge", British Journal of Management, vol. 15, n. 2, pp. 177188.

SURROCA J., TRIBÓ J.A., WADDOCK S. (2010), "Corporate responsibility and financial performance: The role of intangible resources", Strategic Management Journal, vol. 31, n. 5, pp. 463-490.

SWEETLAND S.R. (1996), "Human capital theory: Foundations of a field of inquiry", Review of Educational Research, vol. 66, n. 3, pp. 341-359.

TEECE D.J., PISANO G., SHUEN A. (1997), "Dynamic Capabilities and strategic management”, Strategic Management Journal, vol. 18, n. 7, pp. 509-533.

VAN WEELE M., VAN RIJNSOEVER F.., NAUTA F. (2017), "You can't always get what you want: How entrepreneur's perceived resource needs affect the incubator's assertiveness", Technovation, vol. 59, n. January, pp. 18-33.

VICARI S. (1995), "Verso il resource-based management", in Vicari S. (a cura di) Brand Equity. Il potenziale generativo della fiducia, Egea, Milano.
Cinzia Dessì

Il ruolo delle risorse

Intangibili nella letteratura

manageriale. Principali linee

di ricerca e analisi future 
sinergie Vol. 35, N. 103, 2017

VICARI S., VERONA G. (2000), "La generazione del vantaggio competitivo. Recenti sviluppi e nuove implicazioni per il resource-based management", Finanza, Marketing e Produzione, vol. 18, n. 2, pp. 7-38.

VILLALONGA B. (2004), "Intangible resources, Tobin's q, and sustainability of performance differences”, Journal of Economic Behavior and Organization, vol. 54, n. 2, pp. 205-230.

WERNERFELT B. (1984), "A resource-based view of the firm”, Strategic Management Journal, vol. 5, n. 2, pp. 171-180.

WINDSPERGER J. (2004), "Centralization of franchising networks: evidence from the Austrian franchise sector", Journal of Business Research, vol. 57, n. 12, pp. 1361-1369.

YANNOPOULOS P. (2011), "Defensive and offensive strategies for market success", International Journal of Business and Social Science, vol. 2, n. 13, pp. 1-12.

ZAKERY A., AFRAZEH A. (2017), "Analysing intellectual capital management and growth based on dynamic modelling", Knowledge Management Research and Practice, vol. 15, n. 1, pp. 101-113.

ZAMBON S., MARZO G., SCARPINO E. (2016), "Exploring intellectual capital management in SMEs: an in-depth Italian case study", Journal of Intellectual capital, vol. 17, n. 1, pp. 27-51.

ZYGLIDOPOULOS S.C. (2005), "The impact of downsizing on corporate reputation", British Journal of Management, vol. 16, n. 3, pp. 253-259.

\section{Academic or professional position and contacts}

\section{Cinzia Dessì}

Researcher of Management

University of Cagliari - Italy

e-mail: cdessi@unica.it

\section{Michela Floris}

Researcher of Management

University of Cagliari - Italy

e-mail: micfloris@unica.it

sinergie italian journal of management ISSN 0393-5108 DOI $10.7433 / \mathrm{s} 103.2017 .14$ 